\title{
TASA DE CRECIMIENTO Y DETERIORO DEL MEDIO URBANO EN MÉXICO. UN INTENTO EXPLORATORIO DE LAS RELACIONES EXISTENTES
}

\author{
Ligia Herrera \\ El Colegio de México
}

\section{INTRODUCCIÓN}

UNA DE LAS CARACTERÍsTICAS bien conocidas de la distribución de la población mexicana es su alta concentración urbana. Sin embargo, es bastante menos conocido el hecho de que esta forma de distribución, tal vez por los altos niveles alcanzados en el país, está contribuyendo de manera clara al deterioro del medio ambiente humano.

En este trabajo se intenta presentar este problema, explorándose la relación existente entre deterioro urbano, tasa anual de crecimieno demográfico total y tamaño de la población de las ciudades de 50000 habitantes y más en México. Al hacerlo no se tiene por finalidad ni identificar los factores que han determinado la situación existente ni buscar soluciones que la remedien. El objetivo perseguido es el de exponer la situación tal como se presenta, analizar algunos aspectos de la misma que aún son poco conocidos y poner en evidencia algunas de sus consecuencias en el medio humano.

Se quiere dejar constancia de que no se considera el crecimiento urbano como factor que forzosamente ha de llevar al deterioro del medio. Por el contrario, se reconocen ampliamente las consecuencias de índole positiva que conlleva la urbanización. Se estima, sí, dados el rápido crecimiento de las ciudades y las condiciones económico-sociales en que el mismo opera, que ellas se encuentran en una etapa crítica y que es conveniente llamar la atención sobre las consecuencias negativas de este tipo de crecimiento, tratando al mismo tiempo de esclarecer algunas situaciones básicamente de carácter demográfico en que esos aspectos negativos ocurren.

\section{LA SITUACIÓN ACTUAL}

El deterioro del medio humano urbano ha sido descrito como "un estado disfuncional entre los habitantes de una ciudad y su habitat, que 
tiende a impedir la satisfacción normal y adecuada de sus necesidades afectando por lo tanto negativamente las condiciones de salud física y mental". ${ }^{1}$

En el proceso de desarrollo, la ciudad no industrial tiende a convertirse en un centro industrial-comercial en que se da un notable aumento de la población, producto de un crecimiento natural elevado y del constante flujo de inmigrantes venidos del área rural y de centros urbanos menores. A pesar de su relativo dinamismo económico resulta incapaz de absorber en empleo productivo la creciente mano de obra ni proporcionarle en forma adecuada la gama de servicios urbanos públicos, lo que ha traído graves consecuencias que atañen por igual a las ciudades grandes como a las pequeñas y que se reflejan desfavorablemente en sus respectivos medios humanos. Ciertos aspectos del problema se tornan aún más agudos para las ciudades de reducido tamaño con altas tasas de crecimiento de población dado que, y como características de las pautas y normas de desarrollo existentes, tradicionalmente se da una excesiva concentración de las inversiones, los servicios y las oportunidades de trabajo y de desarrollo cultural en las ciudades más grandes, en detrimento de las demás.

La tendencia creciente que existe en la actualidad de establecer industrias en centros menores como una forma de descongestionar las áreas de mayor concentración, que generalmente son las metropolitanas, torna especialmente grave el problema para aquéllos.

El deterioro del medio humano urbano tiene múltiples facetas de las cuales sólo se destacarán en este trabajo aquellas relacionadas con las deficiencias de ciertos servicios públicos, las que tienden, tal como lo indica la definición antes citada, a impedir la satisfacción normal y adecuada de las necesidades de la población, afectando negativamente sus condiciones de salud física y mental. $\mathrm{El}$ análisis de tales deficiencias, hecho a base de la información que presenta el Censo de Población de 1970, contribuye a esclarecer en forma valiosa las relaciones entre deterioro del medio humano urbano, la tasa de crecimiento y el tamaño de las ciudades que aquí se exploran.

\section{LAS RELACIONES EXISTENTES}

El volumen del Censo de Población de México de 1972,2 relativo a las localidades por entidad federativa y municipio, proporciona datos sobre algunas características de la vivienda de los centros poblados que

1 José Henríquez y Sebastián Riedel, Proceso de metropolización y deterioro urbano, DEPUR-ODEPLAN. Seminario: Proceso de Metropolización en Chile y América Latina, Santiago, 1972 , p. 9.

${ }_{2}$ Dirección General de Estadística, IX Censo General de Población, 1970. Localidades por entidad federativa y municipio con algunas caracteristicas de Población y Vivienda, México, 1973. 
han permitido en este estudio analizar la situación de deterioro de tales características en las ciudades mexicanas que en 1970 tenían una población de 50000 y más habitantes. Teniendo como base la información indicada y complementándola con la población de cada ciudad y su tasa anual de crecimiento demográfico total en el periodo intercensal 1960-1970, se ha elaborado el cuadro 1, que se presenta más adelante. En él, para facilitar el manejo de la información recopilada, las ciudades se han organizado de acuerdo con la magnitud de sus tasas de crecimiento demográfico.

El análisis de los datos ${ }^{3}$ permite llegar a importantes conclusiones sobre las relaciones de que se ocupa este estudio. De esta forma se ha intentado establecer umbrales para las tasas de crecimiento demográfico a partir de los cuales los diferentes servicios públicos de una ciudad dejan de ser satisfactorios.

Con el fin de facilitar el análisis indicado, se ha considerado que cada una de las características de la vivienda deja de ser satisfactoria (o sufre déficit), cuando el $20 \%$ o más de las viviendas de la ciudad carecen de ellas.

La situación de los principales servicios públicos y otras condiciones de vivienda, tal como se deduce de la información recopilada, sería la siguiente:

Agua entubada dentro o fuera del edificio. Un total de 19 centros de los 54 que se analizan en este estudio $(35.2 \%)$ presenta déficit de este servicio, de acuerdo con la clasificación adoptada.

La información del porciento de viviendas sin agua potable y de las tasas de crecimiento de las ciudades, usadas en la gráfica 1, permiten observaciones de interés:

i) No existe una correlación alta entre las dos variables, pero puede apreciarse una tendencia a que las condiciones de abastecimiento de agua sean peores cuando las tasas de crecimiento son altas;

ii) Sólo cuando la tasa de crecimiento demográfico anual es mayor de $4.8 \%$ las ciudades presentan condiciones de déficit $(20 \%$ o más de las viviendas de la ciudad sin este servicio). Existe una excepción: la ciudad de Mérida, capital de un estado económicamente deprimido ( $\mathrm{Yu}$ catán), que con una tasa de crecimiento muy baja, presenta déficit;

iii) A partir del umbral de una tasa de crecimiento anual de $6.4 \%$, todas las ciudades presentan condiciones de déficit. Hay una excepción: Jalapa, capital de un estado de dinamismo económico (Veracruz), que

3 En el análisis se considerarán sólo 54 de las 59 ciudades mexicanas que en 1970 tenían 50000 o más habitantes. Se excluyeron del mismo las ciudades de Poza Rica y Cuernavaca por considerárseles casos de excepción y también los tres centros con población de 1000000 o más habitantes ya que no presentaron déficit (de acuerdo con la clasificación aquí adoptada), en ninguna de las características estudiadas. 
Cuadro 1

MÉxico: algunas Características de la POBLACión y la VIVIENDa EN CIUDADES DE 50000 Y MÁS HABITANTES, 1970

\begin{tabular}{|c|c|c|c|c|c|c|c|c|}
\hline \multirow[b]{2}{*}{ Ciudades } & \multicolumn{7}{|c|}{ Viviends } & $\therefore$ \\
\hline & $\begin{array}{c}\text { Poblacion } \\
=1970\end{array}$ & $\begin{array}{c}\text { Tasa anual do } \\
\text { crecimiento } \\
1960-1970 \\
(\%)\end{array}$ & Wumero & $\begin{array}{c}\text { Sin agua } \\
\text { potablo a } \\
(x)\end{array}$ & $\begin{array}{c}\text { Sin } \\
\text { arenaje } \\
(\%)\end{array}$ & b/ piso de $\begin{array}{c}\text { con } \\
\text { tierra } \\
(\phi)\end{array}$ & $\begin{array}{c}\text { Sin energfa } \\
\text { elfotrica } \\
(\phi)\end{array}$ & $\begin{array}{c}\sin \\
\text { teloviso: } \\
\left(\phi_{0}\right)\end{array}$ \\
\hline 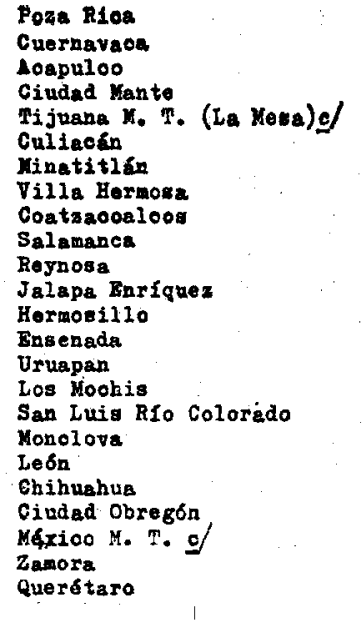 & $\begin{array}{rl}120 & 462 \\
134 & 117 \\
174 & 378 \\
51247 \\
327 \\
1600 \\
167956 \\
68397 \\
99565 \\
69753 \\
61039 \\
137383 \\
122377 \\
176596 \\
77 & 687 \\
82 & 677 \\
67 & 953 \\
49 & 990 \\
78 & 134 \\
364 & 990 \\
257 & 027 \\
114 & 407 \\
859 & 631 \\
57 & 775 \\
112 & 993 \\
-0 & 9 n\end{array}$ & $\begin{array}{r}20.7 \\
14.2 \\
14.0 \\
8.7 \\
8.0 \\
7.3 \\
7.1 \\
6.9 \\
6.7 \\
6.7 \\
6.6 \\
6.6 \\
6.5 \\
6.4 \\
6.3 \\
6.1 \\
6.0 \\
6.0 \\
5.9 \\
5.7 \\
5.6 \\
5.5 \\
5.5 \\
5.5 \\
5.4\end{array}$ & $\begin{array}{rl}21 & 824 \\
25 & 797 \\
30.301 \\
9 & 029 \\
60 & 833 \\
27 & 217 \\
13 & 129 \\
17 & 737 \\
13 & 856 \\
9 & 808 \\
25 & 392 \\
24 & 240 \\
28 & 912 \\
14 & 419 \\
13 & 467 \\
11 & 242 \\
8 & 627 \\
13 & 519 \\
54 & 625 \\
45 & 161 \\
18 & 470 \\
1493 & 248 \\
9 & 241 \\
18 & 096 \\
15 & 241\end{array}$ & $\begin{array}{r}13.8 \\
9.9 \\
26.3 \\
21.3 \\
36.2 \\
23.2 \\
36.6 \\
32.0 \\
34.4 \\
25.0 \\
21.7 \\
12.9 \\
21.3 \\
38.5 \\
16.6 \\
10.1 \\
18.2 \\
27.0 \\
24.7 \\
10.2 \\
13.3 \\
11.6 \\
16.8 \\
5.2 \\
27.1\end{array}$ & $\begin{array}{l}28.9 \\
30.4 \\
45.3 \\
37.9 \\
51.7 \\
48.2 \\
31.8 \\
32.4 \\
31.5 \\
30.2 \\
41.7 \\
29.5 \\
40.5 \\
50.7 \\
31.7 \\
40.3 \\
57.3 \\
52.3 \\
31.9 \\
29.3 \\
33.3 \\
24.4 \\
21.0 \\
25.5 \\
25.7\end{array}$ & $\begin{array}{r}4.6 \\
16.8 \\
30.7 \\
25.5 \\
9.5 \\
33.5 \\
9.8 \\
21.1 \\
6.1 \\
19.1 \\
15.0 \\
13.3 \\
22.2 \\
12.2 \\
29.7 \\
28.0 \\
8.3 \\
8.7 \\
14.1 \\
10.8 \\
23.7 \\
19.7 \\
23.5 \\
10.4 \\
21.8\end{array}$ & $\begin{array}{r}20.2 \\
14.0 \\
33.0 \\
24.8 \\
15.8 \\
17.2 \\
14.1 \\
23.0 \\
5.2 \\
15.7 \\
18.3 \\
11.3 \\
17.8 \\
17.0 \\
17.1 \\
10.3 \\
11.6 \\
13.1 \\
14.2 \\
11.0 \\
12.8 \\
9.0 \\
17.5 \\
21.0 \\
19.1\end{array}$ & $\begin{array}{l}47.3 \\
46.2 \\
55.4 \\
81.1 \\
18.9 \\
55.2 \\
57.3 \\
77.3 \\
56.2 \\
41.2 \\
60.5 \\
49.1 \\
48.5 \\
38.5 \\
82.2 \\
57.7 \\
40.1 \\
77.8 \\
54.6 \\
46.7 \\
52.1 \\
33.4 \\
56.2 \\
41.5 \\
61.9\end{array}$ \\
\hline
\end{tabular}




\begin{tabular}{|c|c|c|c|c|c|c|c|c|}
\hline Saltilio & 767114 & 5.2 & 25 670 & 11.3 & 36.0 & 15.1 & 15.1 & 56.9 \\
\hline Tuxtla Gutierrez & 66851 & 5.1 & 11483 & 17.9 & 32.3 & 27.7 & 19.1 & 73.6 \\
\hline Tepio & 87540 & 5.1 & 15320 & 24.4 & 46.2 & 17.0 & 12.8 & 63.3 \\
\hline Nuevo Laredo & 148867 & 5.0 & 27549 & 13.3 & 31.3 & 15.9 & 14.5 & 42.7 \\
\hline Monterrey M.T. of & 1058130 & 5.0 & 179917 & 5.5 & 25.8 & 11.0 & 90.4 & 38.7 \\
\hline Morolia & 161041 & 5.0 & 9382 & 9.1 & 15.5 & 16.9 & 96.2 & 56.6 \\
\hline Zacateoas & 50225 & 4.9 & 8129 & 23.7 & 34.5 & 22.8 & 28.8 & 57.1 \\
\hline Campeothe & 60506 & 4.9 & 11517 & 32.0 & 48.1 & 16.3 & 17.5 & 65.8 \\
\hline Mazatián & 119553 & 4.8 & 21327 & 10.6 & 33.1 & 16.0 & 14.7 & 53.3 \\
\hline Toluos K.T. of & 144826 & 4.8 & 24035 & 16.3 & 23.1 & 9.9 & 13.7 & 43.8 \\
\hline Ciudad Juároz & 407370 & 4.7 & 74501 & 16.2 & 31.0 & 8.8 & 17.2 & 37.0 \\
\hline Viotoria do Durango & 150541 & 4.6 & 23161 & 13.0 & 25.5 & 19.2 & 16.0 & 58.2 \\
\hline Tampico M.T. $\mathfrak{d}$ & 270414 & $4 \cdot 5$ & 35002 & 11.2 & 22.7 & 10.1 & 14.8 & 50.9 \\
\hline Noxicali & 263498 & $4 \cdot 4$ & 46007 & 17.2 & 50.9 & 12.9 & 8.8 & 27.4 \\
\hline Matamoros & 137749 & 4.2 & 25806 & 16.4 & 39.2 & 14.0 & 19.2 & 60.5 \\
\hline Veraoruz & 214072 & 4.1 & 43900 & 9.1 & 20.1 & 6.5 & 6.3 & 39.1 \\
\hline Tapaohula & 60620 & 4.0 & 11558 & 19.9 & 18.5 & .17 .5 & .17 .5 & 77.2 \\
\hline San Luis Potosi & 230039 & 3.8 & 35660 & 13.7 & 11.8 & 7.9 & 10.2 & 45.9 \\
\hline Aguascalientos & 989277 & 3.8 & 28596 & 5.4 & 17.9 & 10.1 & 13.8 & 46.1 \\
\hline Puebla & 401603 & 3.5 & 73842 & 11.2 & 17.7 & .4 .6 & 12.7 & 41.8 \\
\hline Irapua to & 116651 & 3.5 & 18049 & 8.0 & 19.6 & 14.5 & 18.6 & 46.9 \\
\hline Hidalgo del Parral & 57699 & 3.5 & 9845 & 12.7 & 25.4 & 13.5 & 9.3 & 70.6 \\
\hline Nogales & 52108 & 3.4 & 9382 & 11.7 & 31.7 & 13.0 & 13.3 & 49.2 \\
\hline Oaraca & 99509 & 3.4 & 18786 & 19.5 & 45.7 & 27.2 & 28.1 & 74.2 \\
\hline Colaya & 79977 & 3.2 & 12379 & 4.2 & 19.7 & 12.6 & 12.3 & 36.5 \\
\hline Colima & 58450 & 3.1 & 10015 & 6.2 & 14.1 & 19.4 & 15.4 & 73.1 \\
\hline Orizabs & 92517 & 3.0 & 17653 & 16.7 & 28.3 & 8.2 & 11.2 & 60.2 \\
\hline $\begin{array}{l}\text { Delicias } \\
\text { Paghuon do Soto }\end{array}$ & 52446 & 2.9 & 8754 & 16.4 & 26.8 & 13.8 & 17.5 & 74.9 \\
\hline Paohuo do Soto & 83892 & 2.7 & 14864 & 11.1 & 35.9 & 9.1 & 14.5 & 50.6 \\
\hline $\begin{array}{l}\text { Torrebn-G6mez Palacio X.T. of } \\
\text { M6rida. }\end{array}$ & $\begin{array}{l}302754 \\
212097\end{array}$ & 2.4 & 49451 & 10.3 & 21.7 & 14.2 & 13.0 & 49.0 \\
\hline & & & 37145 & 27.8 & .43 .0 & 12.0 & 13.7 & 48.8 \\
\hline
\end{tabular}

a $\mathrm{Ni}$ dentro ni fuera del edificio.

b Sin drenaje o albañal.

je acuerdo con la clasificación que aparece en El crecimiento urbano de 7 países de América Latina, CELADE, 


\section{Gráfica 1}

MÉxico: CIUDAdes de 50000 Y Más habitantes SEgúN TASA ANUAL DE CRECIMIENTO DEMOGRÁFICO Y VIVIENDAS SIN AGUA POTABLE, 1970ª

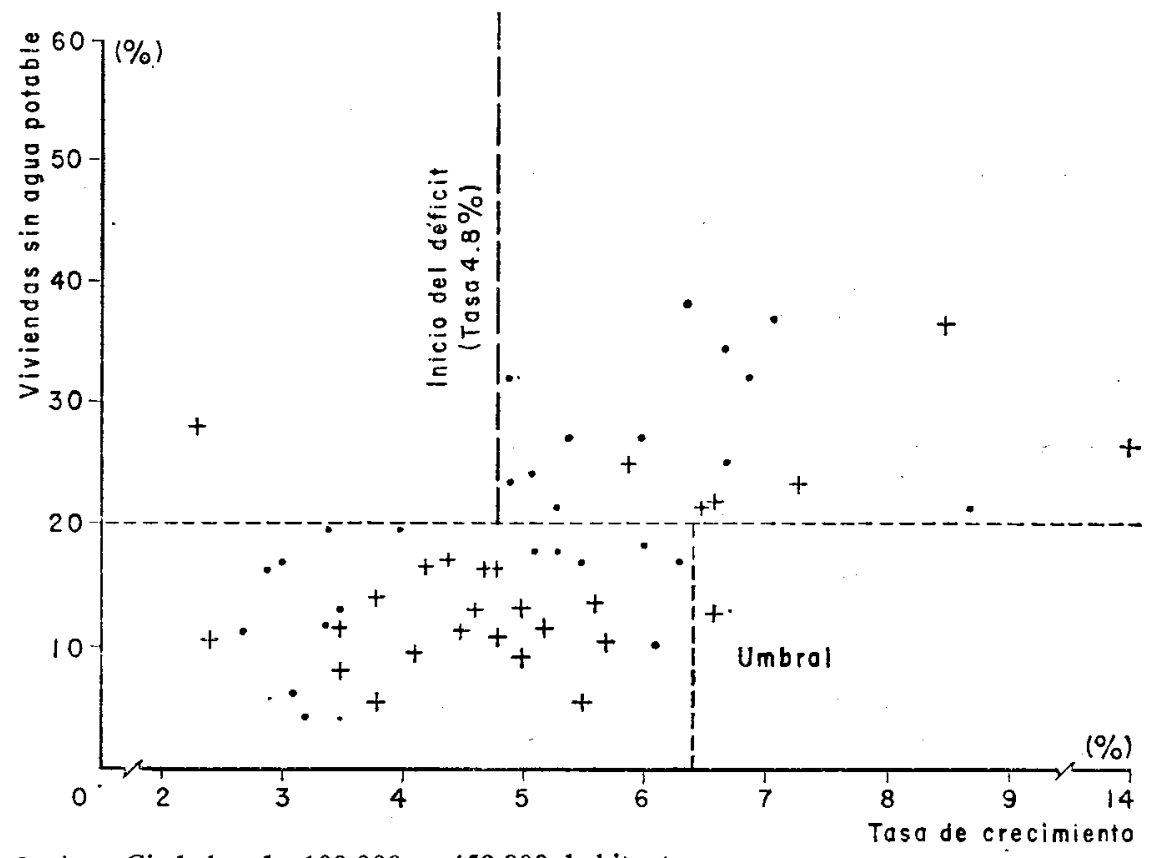

a $+=$ Ciudades de 100000 a 450000 habitantes.

- = Ciudades de 50000 a 99000 habitantes.

con una alta tasa de crecimiento presenta condiciones no deficitarias en este servicio;

iv) En el conjunto de ciudades con déficit de agua privan las ciudades de menor tamaño (50000 a 99000 habitantes).

El análisis de la información contenida en el cuadro 2, ayuda a la comprensión del problema que se estudia. En primer lugar, a nivel del conjunto de las ciudades estudiadas, la relación que existe entre tamaño de la tasa de crecimiento y número de ciudades con déficit de agua entubada es clara. Puede observarse que a las mayores tasas $(6.4 \%$ y más') corresponden el mayor número y el mayor porciento de ciudades con déficit, y que a medida que se reducen las tasas disminuyen también en los respectivos tramos el número y la proporción de ciudades con déficit.

Si el análisis se hace por rango de los tamaños de las ciudades, se podrá observar que los déficit mayores corresponden a las ciudades de menor tamaño (50000 a 99000 habitantes), y que dentro de cada rango el número y la proporción de ciudades deficitarias guardan con las tasas 
Cuadro 2

CIUdades CON déficit de agua potable, SEgún tamaño Y TASA DE CRECIMIENTO DE LA CIUDAD

\begin{tabular}{|c|c|c|c|c|c|}
\hline \multirow{2}{*}{$\begin{array}{l}\text { Tamaño de } \\
\text { 3a ciudada/ }\end{array}$} & \multirow{2}{*}{$\begin{array}{c}\text { Tasa de } \\
\text { crecimiento } \\
\text { demográfico( }(\phi)\end{array}$} & \multicolumn{4}{|c|}{ Ciudades } \\
\hline & & Total & $\frac{\text { Con deficit de }}{\text { Numero }}$ & agrua & $\frac{\text { potablec }}{\%}$ \\
\hline & Total & 54 & 12 & & 35.2 \\
\hline & $\begin{array}{l}6.4 \text { y más } \\
6.3-4.9 \\
4.8 \text { y menos }\end{array}$ & $\begin{array}{l}12 \\
19 \\
23\end{array}$ & $\begin{array}{r}11 \\
7 \\
1\end{array}$ & & $\begin{array}{r}91.7 \\
36.8 \\
4.3\end{array}$ \\
\hline $50-92$ & & 27 & 12 & & 44.4 \\
\hline & $\begin{array}{l}6.4 \text { y más } \\
6.3-4.9 \\
4.8 \text { y menos }\end{array}$ & $\begin{array}{r}6 \\
12 \\
9\end{array}$ & $\begin{array}{l}6 \\
6 \\
-\end{array}$ & & $\begin{array}{r}100.0 \\
50.0 \\
-\end{array}$ \\
\hline $100-450$ & & 27 & 1 & & 25.9 \\
\hline & $\begin{array}{l}6.4 \mathrm{y} \text { mas } \\
6.3-4.9 \\
.4 .8 \mathrm{y} \text { menos }\end{array}$ & $\begin{array}{r}6 \\
7 \\
14\end{array}$ & $\begin{array}{l}5 \\
1 \\
1\end{array}$ & & $\begin{array}{r}83.3 \\
14.3 \\
7.1\end{array}$ \\
\hline
\end{tabular}

a Miles de habitantes.

b Se ha excluido del estudio las ciudades de Poza Rica y Cuernavaca por considerárseles casos de excepción y las de población superior a 1000000 de habitantes que no tienen déficit de agua potable. No existen ciudades con población entre 450000 y 1000000 de habitantes.

c EI $20 \%$ o más de las viviendas sin agua, ni dentro ni fuera del edificio.

de crecimiento la misma relación ya señalada cuando antes se analizaron las ciudades en conjunto. En el caso de las ciudades entre 50000 y 99000 habitantes, aquellas con tasa de crecimiento de $4.8 \%$ o menos, no presentan déficit.

Los análisis anteriores parecen indicar que el déficit en el servicio de agua entubada de una ciudad está estrechamente relacionado con la magnitud de la tasa de crecimiento de su población y que tal relación es más evidente cuando se trata de ciudades pequeñas.

Para simplificar las observaciones anteriores, podrían considerarse como tasas de crecimiento adecuados de la población de una ciudad mexicana para que su servicio de agua entubada se mantenga eficiente, a aquellas inferiores al $5 \%$ anual. De igual modo podría considerarse como umbral de un marcado déficit de este servicio, a aquel que corresponde a tasas de crecimiento de la población mayores del $6 \%$ anual. El tramo que corresponde a tasas de crecimiento entre 5 y $6 \%$, vendría a ser un tramo crítico que indicaría la conveniencia de disminuir el ritmo de crecimiento de la ciudad o de fortalecer su infraestructura en lo que se refiere a agua entubada.

Drenaje. El déficit de drenaje es el más frecuente entre las características de la vivienda de las ciudades que se analizan en este estudio. 
De las 54 ciudades analizadas, $46(85.1 \%$ del total) presentan déficit de drenaje equivalente al $20 \%$ o más de sus viviendas. En estas condiciones se decidió ampliar en este caso el margen que se considera como inadecuado hasta un $30 \%$ de las viviendas. Calificándolas de esta manera, 34 de las 54 ciudades estudiadas resultaron con sistema de drenaje defícitario, lo que correspondió a un $63.0 \%$ del total.

Se utilizó también la información del porciento de viviendas sin drenaje y de las tasas de crecimiento de las ciudades para elaborar una gráfica de correlación de cuya observación podemos llegar a las siguientes conclusiones (véase la gráfica 2 ):

i) No existe una correlación alta entre las dos variables, pero puede apreciarse una tendencia a que las condiciones de drenaje sean menos eficientes cuanto más alta es la tasa de crecimiento anual de las ciudades;

ii) Existen ciudades con déficit aun con tasas de crecimiento bajas (tres con tasas menores al $3.5 \%$ ), pero el mismo se acentúa a partir de tasas de $4.5 \%$ o más;

iii) A partir del umbral de una tasa de crecimiento anual de $6.0 \%$, todas las ciudades sufren déficit de drenaje.

\section{Gráfica 2}

MÉxICO: CIUDADES DE 50000 o Más HabitANTES SEGÚN TASA ANUAL DE CRECIMIENTO DEMOGRÁFICO Y VIVIENDAS SIN DRENAJE, 1970ª

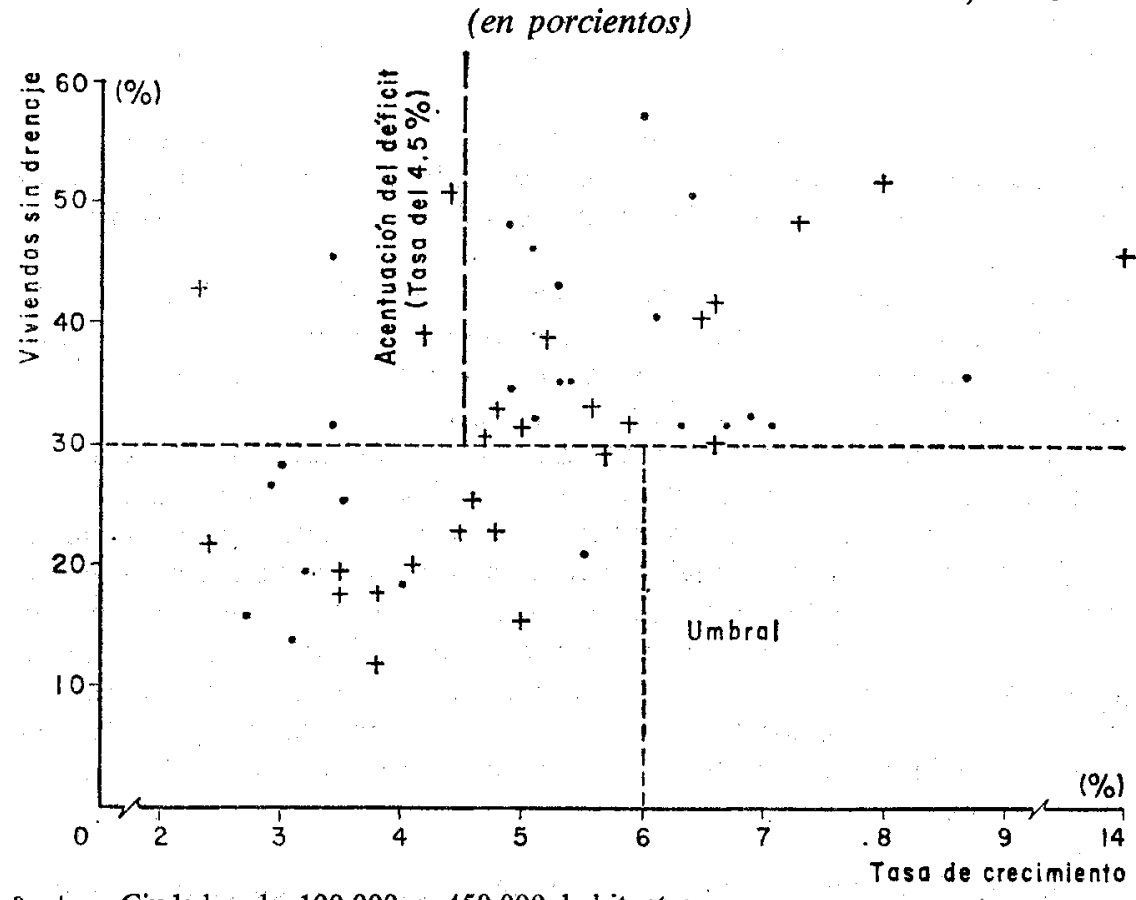

$a+=$ Ciudades de 100000 a 450000 habitantes.

- E Ciudades de 50000 a 99000 habitantes. 
El cuadro 3 contiene información que ayuda a esclarecer el problema estudiado. También en este caso es clara la relación que existe entre tamaño de la tasa de crecimiento y número de ciudades con déficit a nivel nacional. Igualmente puede observarse que a las tasas de crecimiento más altas corresponde el mayor número y porciento de ciudades con déficit y que a medida que las tasas son menores disminuyen el número $\mathrm{y}$ la proporción de ciudades con déficit.

Cuando el análisis se hace por rango de tamaño de las ciudades, se observa que los déficit mayores corresponden a las ciudades más pequeñas con las tasas altas (6.4 o más, y 6.3 a 4.9) y que dentro de cada rango el número y la proporción de ciudades con déficit guardan con las tasas de crecimiento la misma relación señalada al analizarse las ciudades en conjunto. Las ciudades más pequeñas son las que mejores condiciones de drenaje tienen cuando las tasas de crecimiento anual son de $4.8 \% \circ$ menos.

Este análisis parece indicar que el déficit en el servicio de drenaje de una ciudad también está estrechamente relacionado con la magnitud de la tasa de crecimento de su población y que tal relación es más evidente en las ciudades de 50000 a 99000 habitantes. Siendo el déficit de drenaje un daño más generalizado que el de falta de agua entubada debido al alto costo y a la complejidad de la instalación de un servicio de esta naturaleza, parece explicable que el umbral del déficit en este caso se da al nivel de una tasa de crecimiento algo menor que en el del agua entubada.

\section{Cuadro 3}

CIUDADES CON DÉFICIT DE DRENAJE, SEgÚN TAMAÑo Y TASA DE CRECIMIENTO DE LA CIUDAD

\begin{tabular}{|c|c|c|c|c|}
\hline \multirow{2}{*}{$\begin{array}{l}\text { Tamaño de } \\
\text { la ciudada }\end{array}$} & \multirow{2}{*}{$\begin{array}{c}\text { Pasa de } \\
\text { crecimiento } \\
\text { denográfioo( } \not F)\end{array}$} & \multicolumn{3}{|c|}{ Ciudaces } \\
\hline & & Total & $\frac{\text { Con déficit ded }}{\text { Número }}$ & $\frac{\text { drenajog }}{\%}$ \\
\hline & Total & 54 & 34 & 63.0 \\
\hline & $\begin{array}{l}6.0 \text { y mas } \\
5.9-4.9 \\
4.8 \text { y menos }\end{array}$ & $\begin{array}{l}16 \\
15 \\
23\end{array}$ & $\begin{array}{r}16 \\
11 \\
7\end{array}$ & $\begin{array}{r}100.0 \\
73.3 \\
30.4\end{array}$ \\
\hline $50-99$ & & 27 & 19 & 70.4 \\
\hline & $\begin{array}{l}6.0 \text { y más } \\
5.9-4.9 \\
4.6 \text { y menos }\end{array}$ & $\begin{array}{r}10 \\
8 \\
9\end{array}$ & $\begin{array}{r}10 . \\
7 \\
2\end{array}$ & $\begin{array}{r}100.0 \\
87.5 \\
22.2\end{array}$ \\
\hline $100-450$ & & $\underline{27}$ & 15 & 55.6 \\
\hline & $\begin{array}{l}6.0 \mathrm{y} \text { más } \\
5.9-4.9 \\
4.8 \mathrm{y} \text { menos }\end{array}$ & $\begin{array}{r}6 \\
7 \\
14\end{array}$ & $\begin{array}{l}6 \\
4 \\
5\end{array}$ & $\begin{array}{r}100.0 \\
57.1 \\
35.7\end{array}$ \\
\hline
\end{tabular}

a Miles de habitantes.

a Se ha excluido del estudio las ciudades de Poza Rica y Cuernavaca por considerárseles casos de excepción y las de población superior a 1000000 de habitantes que tienen déficit de drenaje. No existen ciudades con población entre 450000 y 1000000 de habitantes.

c El $30 \%$ o más de las viviendas sin drenaje o albañal. 
Pisos de tierra. Para medir el déficit de piso adecuado para las viviendas se consideró como inadecuado al piso de tierra. A su vez, una ciudad fue considerada con déficit de piso adecuado, cuando el $20 \%$ o más de sus viviendas tenían piso de tierra.

En esta ocasión no se analiza, como en las dos características antes estudiadas, un servicio público. La existencia de piso de tierra en la vivienda representaría más bien el nivel económico de sus moradores. Habría que considerar también que tener en la vivienda piso de otro material que no sea tierra, es considerado por las personas símbolo de prestigio y de estatus social. Sin embargo, aunque menos clara que en el caso del agua potable o del drenaje, puede notarse una relación entre tasa de crecimiento anual de la ciudad, tamaño de la misma y déficit de piso adecuado.

Si bien en este caso no puede hablarse de una tasa umbral a partir de la cual todas las ciudades presentan un déficit de piso adecuado, sí es posible señalar, tal como lo indica la gráfica 3, que (excepto en un caso) a partir de una tasa de crecimiento anual de $4.8 \%$ se presenta déficit en casi la mitad del conjunto de ciudades en esta situación (45.2\% del total).

\section{Gráfica 3}

MÉXICO: CIUDADES DE 50000 o MÁS HABITANTES SEGÚN TASA ANUAL DE CRECIMIENTO Y VIVIENDAS CON PISO DE TIERRA, 1970ª

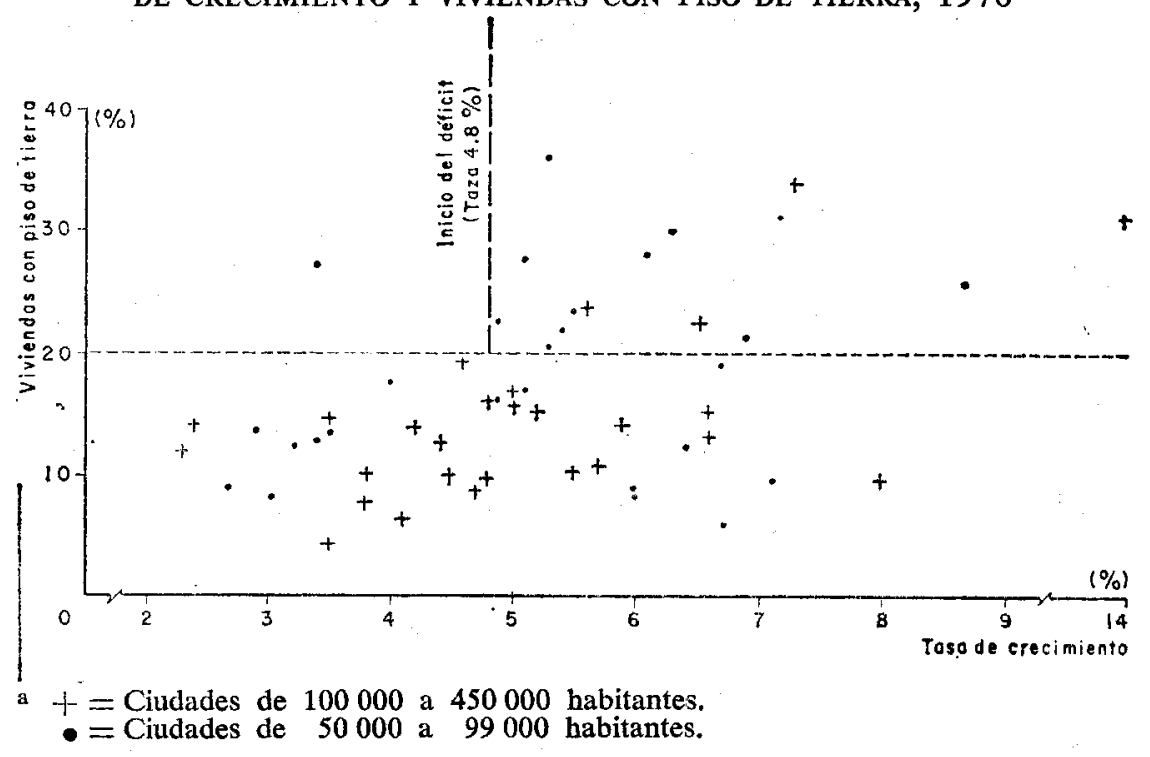

En la misma gráfica puede apreciarse que esta variable es la que presenta más bajos porcientos de déficit y que las ciudades deficitarias son en su mayoría centros con una población entre 50000 y 99000 habitantes. En el cuadro 4 se destaca esa circunstancia. 
Cuadro 4

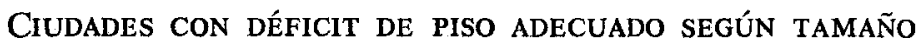

\begin{tabular}{cccc}
\hline Tamaño de la ciudad. & Ciudades & Con pisode tierra \\
\hline Toiles de habitantes) & Total & Fumero \\
\hline Total & 54 & $\frac{15}{11}$ & 27.8 \\
$50-99$ & 27 & 4 & 40.7 \\
$100-450$ & 27 & 14.8 \\
\hline
\end{tabular}

Sin embargo, cuando el análisis se hace teniendo en consideración los rangos de tamaños de ciudades y los de las tasas, como se hizo en los cuadros 2 y 3 , la relación no es suficientemente clara.

En resumen puede expresarse lo siguiente:

i) No existe una tasa umbral a partir de la cual todas las ciudades sufren déficit en la característica que se estudia;

ii) El déficit se inicia a partir de la tasa de crecimiento anual de $4.8 \%$. Más del $45 \%$ de las ciudades con tasas mayores que la apuntada no presentan condiciones adecuadas de piso;

iii) En términos generales, las ciudades de menor tamaño son las que con más frecuencia padecen del déficit señalado.

Energía eléctrica. Ésta es la característica que menos déficit presenta en las ciudades mexicanas. Apenas siete de ellas tuvieron más del $20 \%$ de sus viviendas sin energía eléctrica. Cabría anotar que si seis de estas ciudades tuvieron tasas de crecimiento anual de su población superiores a $4.8 \%$ y que en ningún caso su población alcanzó a los 200000 habitantes, se trata pues de ciudades pequeñas.

La propiedad de un televisor. El censo en referencia presenta también información sobre dos características que pueden ser consideradas como indicadores de niveles de vida; ellas son la existencia de radio y de televisor en la vivienda. Conocidas las implicaciones que los bajos niveles de vida tienen en el deterioro del medio humano, conviene explorar lo que ocurre con estos indicadores.

La propiedad de una radio es hoy día tan universal que en realidad ha perdido valor como medida de nivel de vida. Para que lo mantuviera habría que entrar a clasificar los distintos tipos de radios, cosa prácticamente imposible. En México sólo tres ciudades de las que se analizan tienen más de un $20 \%$ de sus viviendas sin radio.

La situación es diferente cuando se trata de la posesión de un televisor. En el conjunto de ciudades en estudio es frecuente que no exista televisor entre el 35 y el $80 \%$ de las viviendas. Sin embargo, es sólo en las ciudades más pequeñas (50000 a 99000 habitantes) donde esa proporción sobrepasa el $60.0 \%$, tal como puede apreciarse en la gráfica 4 . En la misma puede apreciarse también que si bien no existe una corre- 
lación estrecha entre el tamaño de la ciudad y la proporción de viviendas sin televisor, sí existe una tendencia en el sentido de que a menor tamaño del centro urbano es mayor el déficit de televisores y que este déficit disminuye cuando aumenta el tamaño de la ciudad. En una gráfica del mismo tipo en la que se consideró la existencia de televisores y la tasa de crecimiento de la ciudad no se apreció correlación alta y ni siquiera una tendencia específica. Podría deducirse de lo anterior que el nivel de vida de los habitantes de las ciudades pequeñas, en sentido general pareciera ser menos elevado que el de los habitantes de las ciudades más grandes.

\section{Gráfica 4}

MÉXICO: CIUDADES DE 50000 o MÁS HABITANTES SEGÚN TAMAÑo DE LA CIUDAD Y EXISTENCIA DE TELEVISOR EN LA VIVIENDA, 1970a

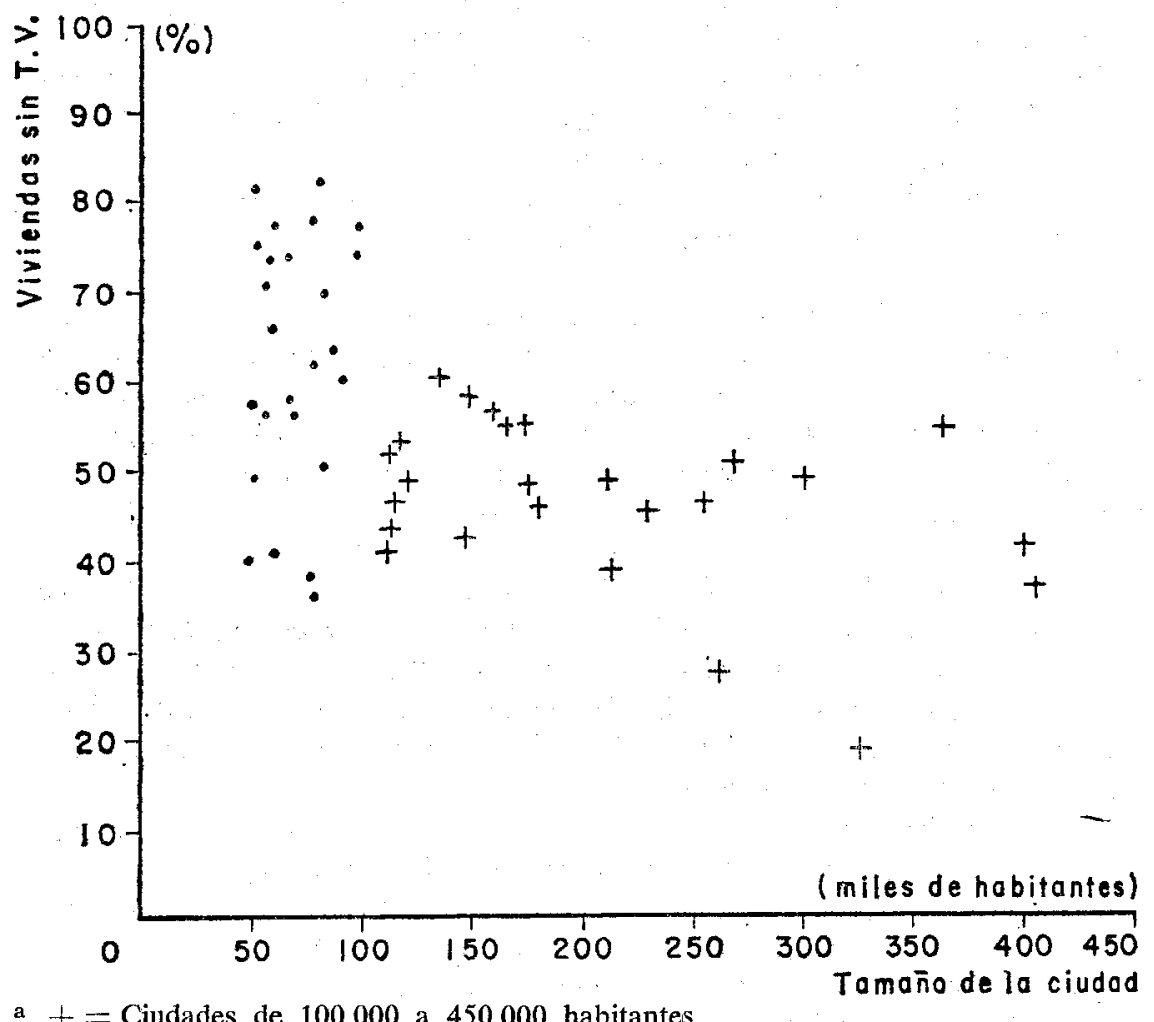

$+=$ Ciudades de 100000 a 450000 habitantes.

Si se agrupan las ciudades que registran déficit en las cuatro principales características estudiadas (agua, drenaje, piso, energía eléctrica) y aquellas que no sufren déficit en ninguna, podrá observarse (véase el cuadro 5) que en el primer caso (cinco ciudades) todas registran, en 
los dos últimos periodos intercensales, aumento en las tasas de crecimiento anual de su población, la que en cuatro casos fue muy importante; cuatro de las cinco que componen el grupo tuvieron una población que no llegó a 100000 habitantes. En cambio, si se examinan las ciudades que no tuvieron déficit de servicios (18), se notará que todas tuvieron tasas de crecimiento anual inferiores a $6 \%$, que seis de ellas sufrieron disminución de sus tasas, ocho tuvieron un débil aumento, y que cuatro registran aumento de cierta consideración que en ningún caso llegó a ser del $2 \%$. Once de estas ciudades tenían una población de más de 100000 habitantes mientras que siete no llegaron a esa cifra; todas estas últimas tuvieron tasas de crecimiento anual de $4 \%$ o menos.

\section{Cuadro 5}

CIUDADES CON Y SIN DEFICIENCIAS, SEGÚN CUATRO CARACTERísticaS Y TASA DE CRECIMIENTO

\begin{tabular}{|c|c|c|c|}
\hline \multirow[t]{2}{*}{ ciudad } & \multirow{2}{*}{$\begin{array}{l}\text { Población } \\
1970\end{array}$} & \multicolumn{2}{|c|}{$\begin{array}{c}\text { Tasa de crecimiento } \\
\text { anual (g) }\end{array}$} \\
\hline & & $1950-1960$ & $1960-1970$ \\
\hline \multicolumn{4}{|l|}{ Con deficiencias } \\
\hline $\begin{array}{l}\text { Acapulco } \\
\text { Ciudad Nante } \\
\text { Villa Hermosa } \\
\text { Ciudad Victoria } \\
\text { Zacatecas }\end{array}$ & $\begin{array}{r}174378 \\
51249 \\
99565 \\
83897 \\
: 50.251\end{array}$ & $\begin{array}{l}5.6 \\
0.7 \\
4.5 \\
4.8 \\
2.7\end{array}$ & $\begin{array}{r}14.0 \\
8.7 \\
6.9 \\
5.3 \\
4.9\end{array}$ \\
\hline \multicolumn{4}{|l|}{ Sin deficiencias } \\
\hline $\begin{array}{l}\text { Chihuahua } \\
\text { Morelia } \\
\text { Toluca } \\
\text { Durango } \\
\text { Tampico } \\
\text { Veracruz } \\
\text { Tapachula } \\
\text { San Luis Potosf } \\
\text { Agrascalientes } \\
\text { Puebla } \\
\text { Irapuato } \\
\text { Hidalgo del Parral } \\
\text { Celaya } \\
\text { Colima } \\
\text { Orizaba } \\
\text { Deliciag } \\
\text { Pachuca } \\
\text { Torreon - Gómez Palacio }\end{array}$ & $\begin{array}{rl}257^{\circ} & 027^{\circ} \\
161 & 040 \\
144 & 826 \\
150 & 541 \\
270 & 414 \\
214 & 072 \\
60 & 620 \\
230 & 039 \\
181 & 277 \\
401 & 603 \\
116 & 651 \\
57 & 619 \\
79 & 977 \\
58 & 450 \\
92 & 517 \\
52 & 446 \\
83 & 892 \\
302 & 754\end{array}$ & $\begin{array}{l}5.6 \\
4.8 \\
3.8 \\
5.0 \\
2.7 \\
3.6 \\
3.3 \\
2.4 \\
3.1 \\
3.2 \\
5.4 \\
2.6 \\
5.5 \\
4.2 \\
2.3 \\
8.1 \\
1.0 \\
3.3\end{array}$ & $\begin{array}{l}5.7 \\
5.0 \\
4.8 \\
4.6 \\
4.5 \\
4.1 \\
4.0 \\
3.8 \\
3.8 \\
3.5 \\
3.5 \\
3.5 \\
3.2 \\
3.1 \\
3.0 \\
2.9 \\
2.8 \\
2.3\end{array}$ \\
\hline
\end{tabular}

\section{Conclusiones}

Del análisis de las cinco características de las ciudades mexicanas estudiadas podría llegarse a conclusiones de interés como son las siguientes:

1. Parece evidente que existe una relación entre la tasa de crecimiento de la población de una ciudad y el deterioro de servicios públicos tales 
como agua potable y drenaje (a tasas altas corresponderían déficit mayores'), apareciendo esta relación en forma menos clara en el caso del servicio de energía eléctrica. Otras características de la vivienda como es la calidad de su piso, también parece afectada cuando las tasas de crecimiento son altas;

2. Para los servicios de agua potable y drenaje existe una tasa de crecimiento umbral a partir de la cual todas las ciudades muestran déficit de los mismos, de acuerdo con la clasificación empleada en este estudio. Tal umbral está representado en el primero de los casos por una tasa de crecimento anual de $6.4 \%$ y en el segundo de $6.0 \%$;

3. Existe una tasa de crecimiento anual umbral, a partir de la cual se inicia el déficit en el servicio de agua potable y en la deficiencia de la calidad del piso de la vivienda. En ambos casos tal tasa es de $4.8 \%$. Esta tasa umbral es menos clara en el caso de los servicios de drenaje que en ocasiones muestran déficit en ciudades con tasas de crecimiento bajas como alrededor de $3.5 \%$. Sin embargo, en este caso es evidente que este déficit se acentúa a partir de tasas de crecimiento anual de $4.5 \%$;

4. Parece evidente que existe una relación entre el tamaño de la ciudad y el deterioro de sus servicios, correspondiendo déficit mayor a las ciudades de menor tamaño (50000 a 99000 habitantes). Cuando estas dos variables son relacionadas con tasas de crecimiento anual, se observa en todas las caracerísticas estudiadas que estas mismas ciudades tienen las peores condiciones cuando las tasas son altas en lo relacionado a agua y drenaje, y las mejores condiciones cuando las tasas son bajas, llegando en el caso del agua potable a no presentar déficit en esa situación;

5. Si se juzga el nivel de vida de los habitantes de una ciudad a partir de la existencia de televisor en la vivienda, parecería que los centros más pequeños tienen, en general, niveles de vida menos eficaces que los de mayor tamaño.

\section{CONSideraciones finales}

Cabría preguntarse si las situaciones indicadas en estas cinco conclusiones se presentan de manera similar en otros países de América Latina. Otras preguntas surgen al tratar de explicarse el porqué de las situaciones aquí descritas. Por ejemplo, qué papel están jugando en ellas los niveles de desarrollo y de urbanización alcanzados por el país, la proporción del presupuesto fiscal destinado a obras públicas, los niveles de ingreso de la población.

No hay dudas sobre la importancia que tiene la tasa de crecimiento de la población en los aspectos estudiados para las ciudades mexicanas. Los resultados obtenidos en este estudio podrían ser de utilidad para la elaboración de medidas de política gubernamental que permitan evitar sus efectos negativos. 